\title{
The dark nature of GRB 051022 and its host galaxy ${ }^{\star}$
}

\author{
A. J. Castro-Tirado ${ }^{1}$, M. Bremer ${ }^{2}$, S. McBreen ${ }^{3}$, J. Gorosabel ${ }^{1}$, S. Guziy ${ }^{1,4}$, T. A. Fakthullin ${ }^{5}$, V. V. Sokolov ${ }^{5}$, \\ R. M. González Delgado ${ }^{1}$, G. Bihain ${ }^{6,7}$, S. B. Pandey ${ }^{1,8}$, M. Jelínek ${ }^{1}$, A. de Ugarte Postigo ${ }^{1}$, K. Misra ${ }^{9}$, R. Sagar ${ }^{9}$, \\ P. Bama ${ }^{10}$, A. P. Kamble ${ }^{11}$, G. C. Anupama ${ }^{12}$, J. Licandro ${ }^{4,13}$, D. Pérez-Ramírez ${ }^{14}$, \\ D. Bhattacharya ${ }^{15,11}$, F. J. Aceituno ${ }^{1}$, and R. Neri ${ }^{2}$
}

\author{
${ }^{1}$ Instituto de Astrofísica de Andalucía (IAA-CSIC), PO Box 3.004, 18.080 Granada, Spain \\ e-mail: ajct@iaa.es \\ 2 Institute de Radioastronomie Milimetrique (IRAM), 300 rue de la Piscine, 38406 Saint-Martin-d'Héres, France \\ 3 Max-Planck-Institut für extraterrestrische Physik, 85748 Garching, Germany \\ ${ }^{4}$ Nikolaev State University, Nikolskaya 24, 54030 Nikolaev, Ukraine \\ 5 Special Astrophysical Observatory (SAO-RAS), Nizhnij Arkhyz, Karachai-Cirkassian Rep. 369167, Russia \\ ${ }^{6}$ Instituto de Astrofísica de Canarias (IAC), Via Láctea s/n, La Laguna, Tenerife, Spain \\ 7 Consejo Superior de Investigaciones Científicas (CSIC), Spain \\ 8 Mullard Space Science Labratory, University College London, Holmbury St. Mary, Dorking, Surrey RH5 6NT, UK \\ 9 Aryabhatta Research Institute of Observational Sciences (ARIES), Manora Peak, Nainital 263 129, India \\ 10 Centre for Research and Education in Science and Technology (CREST), Indian Institute of Astrophysics Shidlaghatta Road, \\ Hosakote 562 114, India \\ 11 Raman Research Institute (RRI), Bangalore 560 080, India \\ 12 Indian Institute of Astrophysics, Koramangala, Bangalore 560 034, India \\ 13 Isaac Newton Group of Telescopes, PO Box 321, 38700 Santa Cruz de la Palma (Tenerife), Spain \\ 14 Departamento de Física, EPS, Universidad de Jaén, Campus Las Lagunillas s/n, A3, 23071 Jaén, Spain \\ 15 Inter-University Centre for Astronomy and Astrophysics, Pune 411007, India
}

Received 14 November 2006 / Accepted 20 August 2007

\section{ABSTRACT}

\begin{abstract}
Aims. We present multiwavelength (X-ray/optical/near-infrared/millimetre) observations of GRB 051022 between $2.5 \mathrm{~h}$ and $\sim 1.15 \mathrm{yr}$ after the event. It is the most intense gamma-ray burst $\left(\sim 10^{-4} \mathrm{erg} \mathrm{cm}^{-2}\right)$ detected by HETE-2, with the exception of the nearby GRB 030329.

Methods. Optical and near infrared observations did not detect the afterglow despite a strong afterglow at X-ray wavelengths. Millimetre observations at Plateau de Bure $(\mathrm{PdB})$ detected a source and a flare, confirming the association of this event with a moderately bright $(R=21.5)$ galaxy.

Results. Spectroscopic observations of this galaxy show strong [O II], H $\beta$ and [O III] emission lines at a redshift of 0.809 . The spectral energy distribution (SED) of the galaxy implies $A_{\mathrm{V}}$ (rest frame) $=1.0$ and a starburst occuring $\sim 25 \mathrm{Myr}$ ago, during which the star-forming-rate reached $\sim 50 M_{\odot} / y r$. In conjunction with the spatial extent $\left(\sim 1^{\prime \prime}\right)$ it suggests a very luminous $\left(M_{\mathrm{V}}=-21.8\right)$ blue compact galaxy, for which we also find $Z \sim Z_{\odot}$. The X-ray spectrum shows evidence of considerable absorption by neutral gas with $N_{\mathrm{H}, \mathrm{X}-\mathrm{ray}}=3.47_{-0.47}^{+0.48} \times 10^{22} \mathrm{~cm}^{-2}$ (rest frame). Absorption by dust in the host galaxy at $z=0.809$ certainly cannot account for the non-detection of the optical afterglow, unless the dust-to-gas ratio is quite different than that seen in our Galaxy (i.e. large dust grains). Conclusions. It is likely that the afterglow of the dark GRB 051022 was extinguished along the line of sight by an obscured, dense star forming region in a molecular cloud within the parent host galaxy. This galaxy is different from most GRB hosts being brighter than $L^{*}$ by a factor of 3 . We have also derived a $S F R \sim 50 M_{\odot} / \mathrm{yr}$ and predict that this host galaxy will be detected at sub-mm wavelengths.
\end{abstract}

Key words. gamma rays: bursts - techniques: photometric - techniques: spectroscopic - X-rays: general - cosmology: observations

\section{Introduction}

The first gamma-ray burst (GRB) with a fading X-ray afterglow and without an optical counterpart was detected by BeppoSAX in Jan 1997 (Feroci et al. 1997; Gorosabel et al. 1998). The number

^ Based on observations taken with the $1.0 \mathrm{~m}$ telescope at ARIES, with the $2.0 \mathrm{~m}$ telescope at Hanle, with the $1.5 \mathrm{~m}$ Carlos Sánchez at Observatorio del Teide, with the $1.5 \mathrm{~m}$ telescope at Observatorio de Sierra Nevada, with the $3.5 \mathrm{~m}$ Telescope at the Spanish-German Calar Alto Observatory, with the $3.5 \mathrm{~m}$ Telescopio Nazionale Galileo, with the $4.2 \mathrm{~m}$ William Herschel telescope, at the Observatorio del Roque de los Muchachos in La Palma, and with the $6.0 \mathrm{~m}$ Bolshoi Azimuthal Telescope at the Special Astrophysical Observatory in Zelenchukskaya. of similar events has continued to increase during the Afterglow Era. Dark GRBs seem to constitute a significant fraction of the GRB population (e.g. Filliatre et al. 2006). Although in these cases where no optical/near infrared (nIR) afterglows have been detected, transient X-ray and radio emission has pinpointed the parent galaxies where the bursts occurred. About 48\% (34/71) of GRBs that were localised by Swift/XRT during the first year of the mission do not show an optical (or near-IR) afterglow, despite deep searches (down to 21-22 mag) performed for nearly all events within $24 \mathrm{~h}$.

Proposed explanations include obscuration, a low-density environment, intrinsic faintness and a high-redshift location (also discussed by Jakobsson et al. 2004; Rol et al. 2005; 
Table 1. Journal of optical and near-infrared (nIR) frames taken on the GRB 051022 field.

\begin{tabular}{lcccc}
\hline \hline $\begin{array}{l}\text { Date of exposure UT } \\
\text { (mid exposure) }\end{array}$ & $\begin{array}{c}\text { Telescope/ } \\
\text { Instrument }\end{array}$ & $\begin{array}{c}\text { Filtre/ } \\
\text { Grism }\end{array}$ & $\begin{array}{c}\text { Exposure time } \\
\text { (seconds) }\end{array}$ & $\begin{array}{c}\text { Limiting } \\
\text { magnitude }\end{array}$ \\
\hline 22 Oct. 05, 16:18 & 2.0HCT (HFOSC) & $R$ & 600 & 22.5 \\
22 Oct. 05, 17:00 & 2.0HCT (HFOSC) & $I$ & 600 & 21.8 \\
22 Oct. 05, 17:20 & 1.0ST (CCD) & $R$ & 900 & 20.8 \\
24 Oct. 05, 20:45 & 1.5OSN (CCD) & $B$ & 1800 & 23.0 \\
24 Oct. 05, 22:15 & 1.5OSN (CCD) & $V$ & 720 & 22.5 \\
24 Oct. 05, 19:40 & 1.5OSN (CCD) & $R$ & 120 & 22.2 \\
24 Oct. 05, 19:43 & 1.5OSN (CCD) & $I$ & 120 & 21.3 \\
26 Oct. 05, 20:00 & 1.5OSN (CCD) & $U$ & 3000 & 22.9 \\
31 Oct. 05, 03:30 & 1.5OSN (CCD) & $U$ & 6000 & 23.4 \\
07 Nov. 05, 22:47 & 4.2WHT (ISIS) & R300B & 5400 & - \\
07 Nov. 05, 22:47 & 4.2WHT (ISIS) & R300B & 1800 & - \\
07 Nov. 05, 23:24 & 4.2WHT (ISIS) & R300R & 3600 & - \\
12 Dec. 06, 17:02 & 6.0BTA (SCORPIO) & VPHG400 & 7200 & - \\
23 Oct. 05, 01:56 & 1.5TCS (CAIN-2) & $K_{S}$ & 1800 & 18.0 \\
23 Oct. 05, 02:42 & 1.5TCS (CAIN-2) & $J$ & 1800 & 19.0 \\
23 Oct. 05, 23:30 & 1.5TCS (CAIN-2) & $H$ & 1800 & 18.5 \\
24 Oct. 05, 00:20 & 1.5TCS (CAIN-2) & $K_{S}$ & 1800 & 18.0 \\
20 Nov. 05, 20:52 & 3.5TNG (NICS) & $J$ & 1200 & 20.0 \\
20 Nov. 05, 21:28 & 3.5TNG (NICS) & $H$ & 1200 & 19.5 \\
14 Oct. 06, 00:39 & 3.5CAHA (OMEGA2000) & $H$ & 2400 & 20.0 \\
\hline
\end{tabular}

Fynbo et al. 2001). The first possibility could be due to: i) a high column density of gas around the progenitor like a dusty, clumpy medium in a giant molecular cloud (GMCs, Lamb 2001) or ii) to dust in the host galaxy at larger distances which can account for only $\sim 10 \%$ of the events (discussed in Piro et al. 2002). The occurrence of a burst in a low-density ambient medium (Taylor et al. 2000) will result in a very dim afterglow, although this seems unlikely due to the accepted association between longduration GRBs and core-collapse supernova whose progenitors would not have had time to travel too far from their birth places in star-forming regions. The high- $z$ case, in which the Ly- $\alpha$ forest emission will affect the optical band, it is only applicable for $\geq 10 \%$ of the events (see Gorosabel et al. 2004).

Therefore it is essential to find new potential dark GRBs and to study whether the darkness is due to the obscuration scenario (the a-priori most plausible scenario for the reasons given above). Thus, the bright GRB 051022 constituted a perfect case study. It was discovered by HETE-2 on 22 Oct. 2005 (Olive et al. 2005). The burst started at 13:07 UT and lasted for $\approx 200 \mathrm{~s}$, putting it in the "long-duration" class of GRBs (Tanaka et al. 2005). It was also observed by Mars Odyssey and Konus/WIND (Hurley et al. 2005). It had a fluence of $(2.20 \pm 0.02) \times 10^{-5} \mathrm{erg} \mathrm{cm}^{-2}$ in the $2-30 \mathrm{keV}$ range and $(1.40 \pm$ $0.02) \times 10^{-4} \mathrm{erg} \mathrm{cm}^{-2}$ in the $30-400 \mathrm{keV}$ energy band, making it the highest fluence event detected by HETE-2 in its 6-yr lifetime, with the sole exception of the nearby GRB 030329 (Ricker 2005). The average spectrum of the prompt emission was best fit by an absorbed Band model with $\alpha=1.01_{-0.03}^{+0.02}, \beta=1.95_{-0.14}^{+0.25}$, $E_{\text {peak }}=213 \pm 18 \mathrm{keV}$ with $N_{\mathrm{H}}=\left(1.51_{-0.50}^{+0.53}\right) \times 10^{22}$ atoms cm $\mathrm{cm}^{-2}$ (Nakagawa et al. 2006). Golenetskii et al. (2005) report the best fit to the Konus data is a power law with exponential cutoff model with alpha $=1.176 \pm 0.038$ and $E_{\text {peak }}=510_{-33}^{+37} \mathrm{keV}$ in the $20 \mathrm{keV}-2 \mathrm{MeV}$ energy range, a higher value than derived by HETE-2. The prompt dissemination $(55.0 \mathrm{~s})$ of the GRB position by HETE-2 enabled rapid responses by automated and robotic telescopes like ROTSE-III (Schaefer et al. 2005) and ART (Torii et al. 2005), although no prompt optical afterglow was detected. Swift slewed and started data acquisition (about $3.5 \mathrm{~h}$ after the event) and fading X-ray emission was detected by the XRT, which can be considered as the first clear detection of the afterglow from GRB 051022 (Racusin et al. 2005a). This result triggered a multiwavelength campaign at many observatories aimed at detecting the afterglow at other wavelengths.

Here we report the results of the multiwavelength observations carried out, from millimetre wavelengths to the X-ray band, for both the afterglow and for the host galaxy, and we discuss the implications of the study for dark GRBs.

\section{Observations and data reduction}

\subsection{Optical and near-IR observations}

Target of opportunity (ToO) observations in the optical were started $2.9 \mathrm{hr}$ after the event using the $1.0 \mathrm{~m}$ telescope (+CCD) at ARIES and the $2.0 \mathrm{~m}$ telescope (+ HFOSC camera) at Hanle (near Himalaya in India). Additional optical observations were made at $1.5 \mathrm{~m}$ telescope (+ CCD) at Observatorio de Sierra Nevada in Granada (Spain). Near-IR (nIR) observations were started at the $1.5 \mathrm{~m}$ Carlos Sánchez telescope (+ CAIN-2) at Observatorio del Teide in Tenerife (Spain) and with the $3.5 \mathrm{~m}$ Telescopio Nazionale Galileo (+ NICS) at Observatorio del Roque de Los Muchachos in La Palma (Spain). Spectroscopic observations of the potential host galaxy were conducted at the $4.2 \mathrm{~m}$ William Herschel Telescope at La Palma on 7 Nov. 2005, using ISIS with the blue arm centred at $4500 \AA$ and the R300B grating (3200-5300 $\AA$, dispersion $0.86 \AA /$ pix) and the red arm centred at $6450 \AA$ and the R300R grating (5300-7600 $\AA$, dispersion $0.84 \AA /$ pix). A second spectrum was obtained with the red arm centred at $8650 \AA$ and the R300R grating (7500$9800 \AA$, dispersion $0.86 \AA /$ pix). A third spectrum was obtained at the $6.0 \mathrm{~m}$ Bolshoi Azimuthal Telescope (BTA) in Caucasus on 12 Dec. 2006, using SCORPIO with the VPHG400 holographic grism (3500-9500 $\AA, 15 \AA$ resolution). The observation log is presented in Table 1.

In order to determine the magnitudes in all our optical and nIR images, we used aperture photometry with the DAOPHOT 


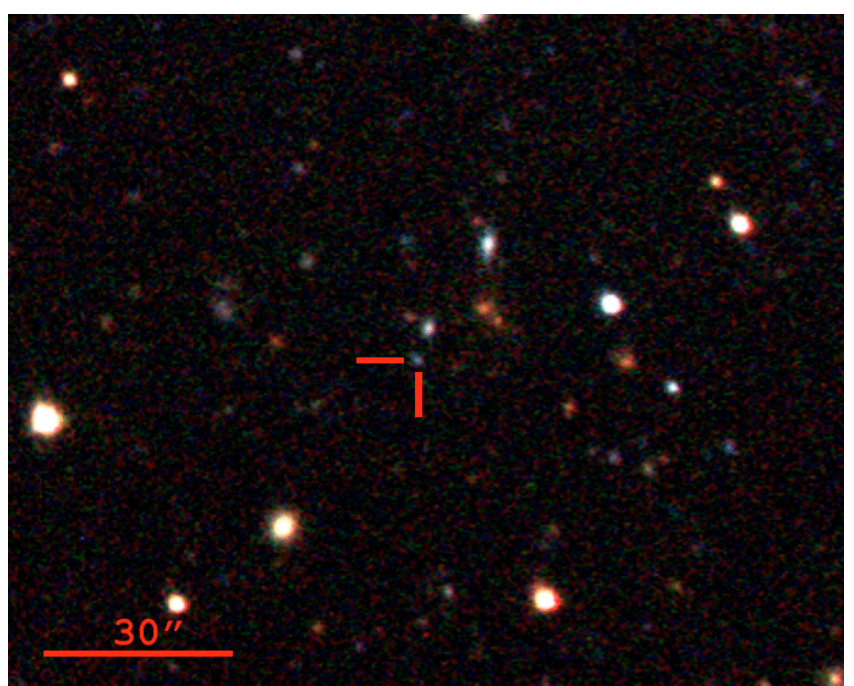

Fig. 1. The $B V R$-band composite image of the GRB 051022 field taken at the $1.5 \mathrm{~m}$ OSN on 26 Oct. 2005 . The potential host galaxy is the bluest object in the field, close to the centre of the image. The field is 2 '. $1 \times 1$.7 with North up and East to the left.

routine under IRAF ${ }^{1}$. The potential host galaxy was revealed in the optical (Fig. 1). The optical field was calibrated using the calibration provided by Henden (2005). The nIR images were calibrated using the 2MASS Catalogue.

The spectroscopic observations were reduced under IRAF following the standard procedure to correct the frames from bias and flatfield. The spectra are also calibrated in wavelength and flux, making use of a spectrophotometric standard star (using a $30^{\prime \prime}$ mask) and taking into account a correction factor for the slit losses.

\subsection{Millimetre observations}

Additional $\mathrm{mm}$ observations were obtained at the Plateau de Bure Interferometer (PdBI) as part of an on-going ToO programme. The PdBI observed the source on Oct. 23, 24, 28, 30 and Nov. 1, in a compact 6 and 5 antenna D configuration. The data reduction was done with the standard CLIC and MAPPING software distributed by the Grenoble GILDAS group, the flux calibration is relative to the carbon star MWC349. Amplitude and phase calibration were relative to the quasar $3 \mathrm{C} 454.3$. The observing log is presented in Table 2 .

\subsection{X-ray observations}

The XRT on Swift began to observe GRB $051022 \sim 3.5 \mathrm{~h}$ after the trigger and detected a decaying X-ray source in the Swift/XRT field at position $\mathrm{RA}(\mathrm{J} 2000)=23^{\mathrm{h}} 56^{\mathrm{m}} 4.1^{\mathrm{s}}$, $\operatorname{Dec}(\mathrm{J} 2000)=+19^{\circ} 36^{\prime} 25^{\prime \prime}$ (Racusin et al. 2005a). The XRT observations are in Photon Counting (PC) mode and were reduced using the standard xrtpipeline (version 0.10.4) for XRT data analysis software ${ }^{2}$ using the most recent calibration files. The spectral data were analysed with the XSPEC version 11.3 (Arnaud 1996).

The first two observations show evidence of mild pile up and an annular source region rather than a circular one was used in

\footnotetext{
${ }^{1}$ IRAF is distributed by the NOAO, which are operated by USRA, under cooperative agreement with the US NSF.

2 http://swift.gsfc.nasa.gov/docs/software/ lheasoft/download.html
}

both of these cases (for a detailed discussion of PC pile up in XRT see Vaughan et al. 2006). The radius of the affected inner annulus was determined by fitting the point spread function to the data and selecting regions where the data are are well fit by the point spread function. Inner annuli regions of $10^{\prime \prime}$ and $5^{\prime \prime}$ were excluded in this manner for orbit 1 and 2 respectively. The exposure map correction was used was to account for the presence of bad columns close to the centre of the source image. In addition, the count rate was manually corrected for the loss of counts incurred by the use of an annular extraction region for the pile up correction.

\section{Results and discussion}

The afterglow was detected at X-ray and millimetre wavelengths, but not in the optical or near infrared bandpasses. The latter non-detections are in agreement with the upper limits reported by ROTSE-III (Schaefer et al. 2005) and ART (Torii et al. 2005).

\subsection{No optical/nIR afterglow}

The Swift/XRT detected the X-ray afterglow for GRB 051022 (Racusin et al. 2005a), allowing us to promptly identify two objects (dubbed "A" and "B", adopting the naming convention introduced by Castro-Tirado et al. 2005) in the surroundings of the XRT X-ray error box. Only object "B" was located inside the box while "A" was just 1.5 arcsec outside. Neither of the two objects were found to be varying in our images, in agreement with other early time reports by Nysewander et al. (2005) or Burenin et al. (2005).

In order to estimate the optical and nIR limiting magnitudes of the possible underlying transient at these wavelengths, we simulated a point source underlying in the host galaxy, making use of the PSF in nearby stars in the field in both the deep $R_{\mathrm{c}}$ and $K_{\mathrm{S}}$-band images, and determined the limiting magnitude at which the simulated point source would remain detected at a $3 \sigma$ level by our detection software. These values, $R=21.5$ and $K_{\mathrm{S}}=18.0$, are used hereafter.

\subsection{Millimetre afterglow: flaring activity ?}

In order to detect the elusive afterglow, mm observations were conducted at PdBI $33 \mathrm{hr}$ after the burst. The afterglow was successfully detected, with a flux density of $1.14 \pm$ $0.28 \mathrm{mJy}$ at $90 \mathrm{GHz}$, at a position $\mathrm{RA}(\mathrm{J} 2000)=23^{\mathrm{h}} 56^{\mathrm{m}} 04.15^{\mathrm{s}}$, $\operatorname{Dec}(\mathrm{J} 2000)=+19^{\circ} 36^{\prime} 25^{\prime \prime}{ }^{\prime} 1\left( \pm 00^{\prime} 6\right)$. The source was coincident, within errors, with the above-mentioned source B, which was found to be extended on images taken by Berger \& Wyatt (2005). Thus, the position of the millimetre afterglow position is superimposed on a bright optical/nIR potential host galaxy. The $\mathrm{mm}$ afterglow faded substantially in $24 \mathrm{~h}$ and the object was not detected on 24 or 28 October. Another detection was obtained on 31 October (see Fig. 2), thus confirming the trend observed at $4.8 \mathrm{GHz}$ (Fig. 1 of Rol et al. 2007). Such a mm reflaring at late epoch might be caused by an energy injection episode but the lack of detailed observations at other wavelengths prevents confirmation.

We also unsuccessfully searched for line emission around the $3 \mathrm{~mm}$ wavelength and therefore "cut" the spectral information of the $90 \mathrm{GHz}$ observations into large velocity bins (100, $200 \mathrm{~km} \mathrm{~s}^{-1}$ ) to check if there is a line profile (which should have 
Table 2. Journal of millimetre (mm) observations of the GRB 051022 field.

\begin{tabular}{lccrcc}
\hline $\begin{array}{l}\text { Date of 2005 UT } \\
\text { (start time - end time) }\end{array}$ & Configuration & \multicolumn{1}{c}{$\begin{array}{c}\text { Flux } \\
(\mathrm{mJy} \sigma)\end{array}$} & $\begin{array}{r}\text { Frequency } \\
(\mathrm{GHz})\end{array}$ & $\begin{array}{c}\text { Beam } \\
(\operatorname{arcsec})\end{array}$ & $\begin{array}{c}\text { Position angle } \\
\left({ }^{\circ}\right)\end{array}$ \\
\hline 23.Oct. 20:42-23.Oct. 23:27 & 6Dp & $1.14 \pm 0.28$ & 90.808 & $8.7 \times 4.0$ & -103 \\
& & $0.02 \pm 1.68$ & 217.827 & $8.9 \times 1.7$ & +64 \\
24.Oct. 19:17-25.Oct. 01:44 & 6Dp & $0.08 \pm 0.17$ & 86.243 & $9.1 \times 4.6$ & +79 \\
& & $-2.42 \pm 0.85$ & 232.032 & $4.7 \times 1.6$ & +73 \\
28.Oct. 20:44-29.Oct. 01:28 & 6Dp & $-0.33 \pm 0.44$ & 86.243 & $9.2 \times 3.8$ & -96 \\
& & $1.81 \pm 3.40$ & 232.032 & $2.5 \times 1.8$ & +57 \\
30.Oct. 15:27-30.Oct. 18:20 & 5Dp & $3.48 \pm 1.05$ & 90.229 & $10.7 \times 3.5$ & -57 \\
01.Nov. 15:54-01.Nov. 21:39 & 5Dp & $-0.50 \pm 0.26$ & 86.243 & $8.0 \times 3.8$ & -69 \\
& & $0.55 \pm 1.35$ & 221.501 & $3.0 \times 1.4$ & -63 \\
\hline
\end{tabular}

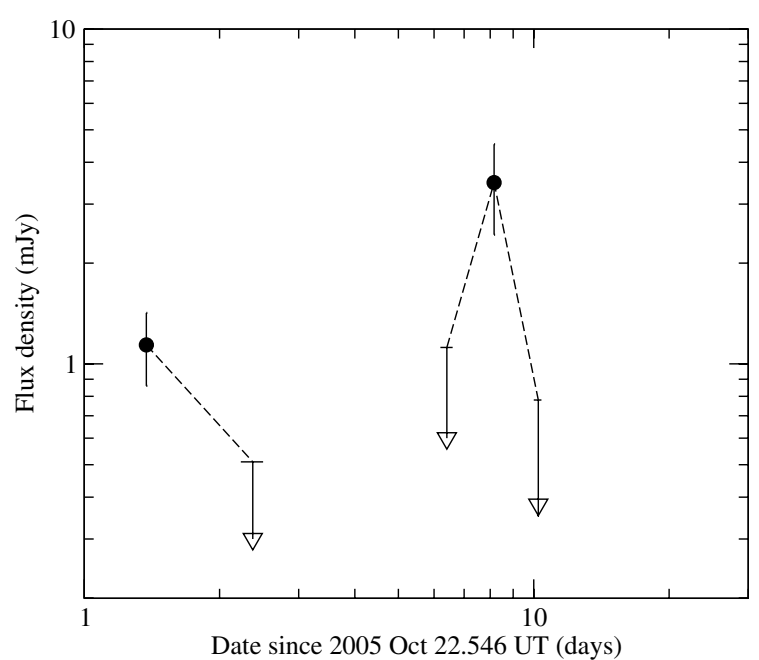

Fig. 2. The $3 \mathrm{~mm}$ wavelength afterglow lightcurve of GRB 051022 obtained at PdBI. Detections were obtained at $90 \mathrm{GHz}$ (filled circles) whereas only upper limits were obtained at $86 \mathrm{GHz}$.

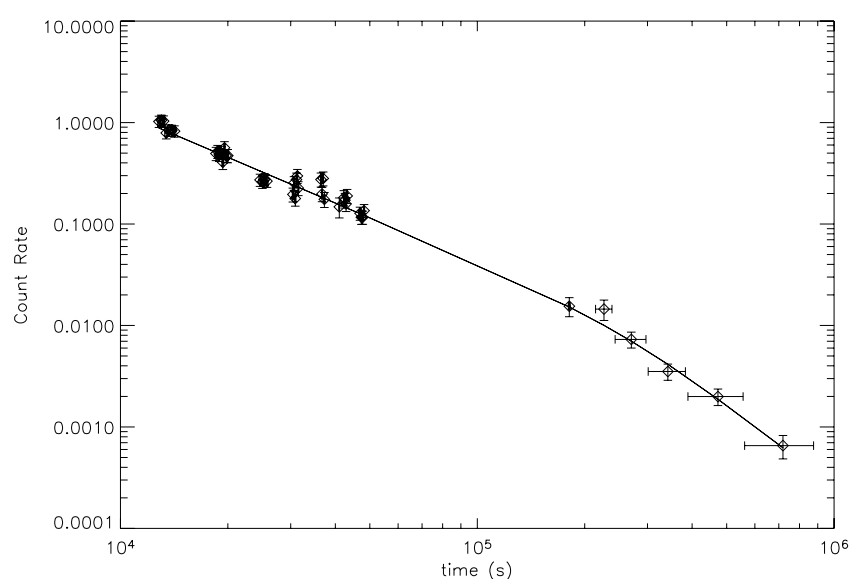

Fig. 3. The Swift/XRT light curve of GRB 051022. A break at $\sim 2 \times 10^{5} \mathrm{~s}$ is apparent on the data.

a signal-to-noise ratio $\geq 3$, because the average over the whole continuum band would mix the line with noise and weaken it).

\subsection{The X-ray afterglow}

The X-ray light curve of GRB 051022 in the energy range 0.3 to $10 \mathrm{keV}$ is shown in Fig. 3. The data is best fit by a broken power law with pre-break index, $\alpha_{X 1}=1.50 \pm 0.07$, post-break index $\alpha_{X 2}=2.47 \pm 0.40$ and a break at $216 \pm 55 \mathrm{ks}$ using a smoothing factor of 10 . The fit is roughly consistent with the values reported

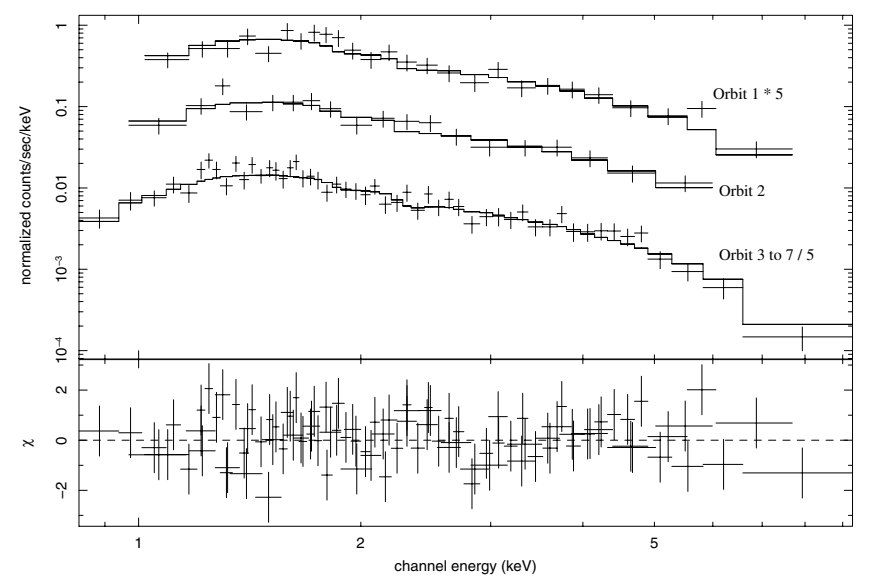

Fig. 4. Pre-break spectra of GRB 051022 including orbits 1, 2 and 3 to 7 separately. The spectra are offset as indicated for clarity of presentation.

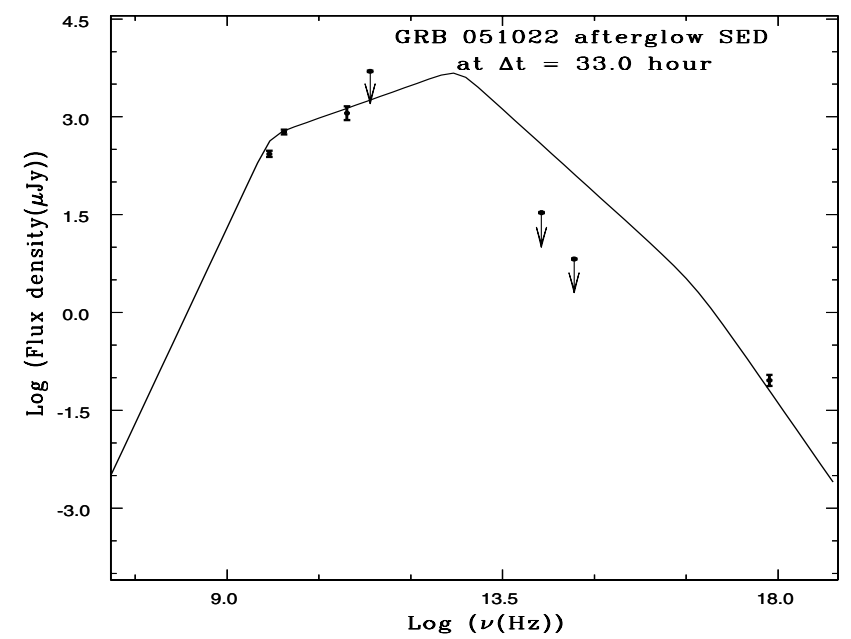

Fig. 5. The SED of the GRB 051022 afterglow at $T_{0}+33 \mathrm{~h}$ (prior to the 2.9 day break time reported by Racusin et al. 2005). We consider $p=$ 2.6 , assuming the slow-cooling regime. We have also included the radio detections at the WSRT and VLA (van der Horst et al. 2005; Cameron \& Frail 2005), as well as the $220 \mathrm{GHz}$ and optical and near-IR upper limits reported in this paper. The initial parameters given at $T_{0}+0.06$ days are $v_{a}=5.1 \times 10^{9} \mathrm{~Hz}, v_{m}=6.5 \times 10^{14} \mathrm{~Hz}, v_{c}=2.1 \times 10^{17} \mathrm{~Hz}$ and $F_{v, \max }=5.5 \mathrm{mJy}$.

by Racusin et al. (2005b) who obtain a pre-break power law index of $1.33 \pm 0.07$, a break at $2.9 \pm 0.2$ days $(250 \pm 17 \mathrm{ks})$ after which the power-law index changed to $3.6 \pm 0.4$ and the differences can likely be attributed to the availability of new software 
and responses. Rol et al. (2007) report an earlier break time of $110_{-23}^{+21} \mathrm{ks}$ based on the Swift/XRT and Chandra data.

The spectra shown in Fig. 4 were extracted individually for orbit 1,2 and 3 to 7 and were fit simultaneously. The data have 20 counts per bin. The pre-break X-ray spectra can be fit by a photon index $\Gamma=2.25 \pm 0.15$ and a column density of $\left(0.85_{-0.11}^{+0.12}\right) \times 10^{22} \mathrm{~cm}^{-2}$ with $\chi^{2} /$ d.o.f. $=81 / 93$. A value of $3.47_{-0.47}^{+0.48} \times 10^{22} \mathrm{~cm}^{-2}$ was obtained for the extra absorption by a cold neutral gas within the host galaxy at redshift 0.809 , in addition to the absorption within our own galaxy which is $4.09 \times$ $10^{20} \mathrm{~cm}^{-2}$ (Dickey \& Lockman 1990). We find no evidence for spectral evolution in the X-ray spectrum of GRB 051022. This analysis is in agreement with the XRT results obtained by Butler et al. (2005) and and Rol et al. (2007). Nakagawa et al. (2006) derive a higher value of the column density of $8.8_{-1.8}^{+1.9} \times 10^{22} \mathrm{~cm}^{-2}$ (rest frame) in the prompt emission, and Rol et al. (2007) point out that this could indicate a change in absorption between the early HETE-2 observations and those of the XRT.

\subsection{Spectral energy distribution modelling}

We have determined the spectral energy distribution (SED) for GRB 051022 at $T_{0}+33 \mathrm{~h}$ at time of the PdB observations (Fig. 5). The data are well fit by a jet model with $p=2.6 \pm 0.2$, as $\alpha=(3 p-2) / 4$ and $\beta=p / 2$ for pre-break (isotropic emission) and $v_{\mathrm{X}}>v_{c}$ derived from the $\mathrm{X}$-ray data and assuming the slowcooling regime (Sari et al. 1998). We cannot distinguish whether the external medium is ISM $(\rho=$ constant $)$ or a stellar wind profile $\left(\rho \propto r^{-2}\right)$. We have also included the radio detections at the WSRT and VLA (van der Horst et al. 2005; Cameron \& Frail 2005).

\subsection{The obscuring medium}

GRB 051022 is located undoubtely in the dark GRB locus of the $F_{\text {opt }}-F_{\text {X-ray }}$ diagram of Jakobsson et al. (2004). Clearly the reason that the afterglow was not detected either at optical or at nIR wavelengths is due to obscuration along the line of sight. The spectral energy distribution can be used to estimate the amount of extinction by dust at optical wavelengths and predicts a magnitude $R \sim 18.3$ at $T_{0}+33 \mathrm{~h}$. The $R$ band upper limit from our observations is $21.5 \mathrm{mag}$ and therefore we infer a considerable extinction toward GRB 051022, with a lower limit $A_{\mathrm{R}}=3.2 \mathrm{mag}$ in the observer frame. This is equivalent to $A_{\mathrm{V}}=1.7 \mathrm{mag}$ in the rest frame at $z=0.809$ (see next subsection) from which an equivalent Hydrogen column density at rest frame of $N_{\mathrm{H}, \mathrm{opt}} \geq 0.4 \times 10^{22} \mathrm{~cm}^{-2}$ is derived, assuming the relationship $A_{V}=0.56 \times N_{\mathrm{H}}\left(10^{21} \mathrm{~cm}^{-2}\right)+0.23$ of Predehl \& Schmitt (1995). A value of $N_{\mathrm{H}, \mathrm{X} \text {-ray }}=\left(3.47_{-0.47}^{+0.48}\right) \times 10^{22} \mathrm{~cm}^{-2}$ (rest frame) was derived from the X-ray data assuming the absorbing material being in a neutral cold state. Therefore, the ratio of $N_{\mathrm{H}, \mathrm{X} \text {-ray }} / N_{\mathrm{H} \text {,opt }}$ is less than 9 .

Comparable values of $\mathrm{N}_{\mathrm{H}, \mathrm{X} \text {-ray }}$ (rest frame) were found in other GRBs like GRB 980703 (Galama \& Wijers 2001) and GRB 050401 (Campana et al. 2006). Substantially higher values were found in another two dark GRB host galaxies GRB 970828 (Djorgovski et al. 2001) and GRB 000210 (Piro et al. 2002), assuming the gas-to-dust ratio is compatible with the Galactic value.

Is it possible that the absorption took place in the circumburst environment of the GRB by the ISM of the host galaxy? The value of $A_{\mathrm{V}}=1.0$ derived from the host galaxy SED (see Sect. 3.4) cannot account for the properties of this event

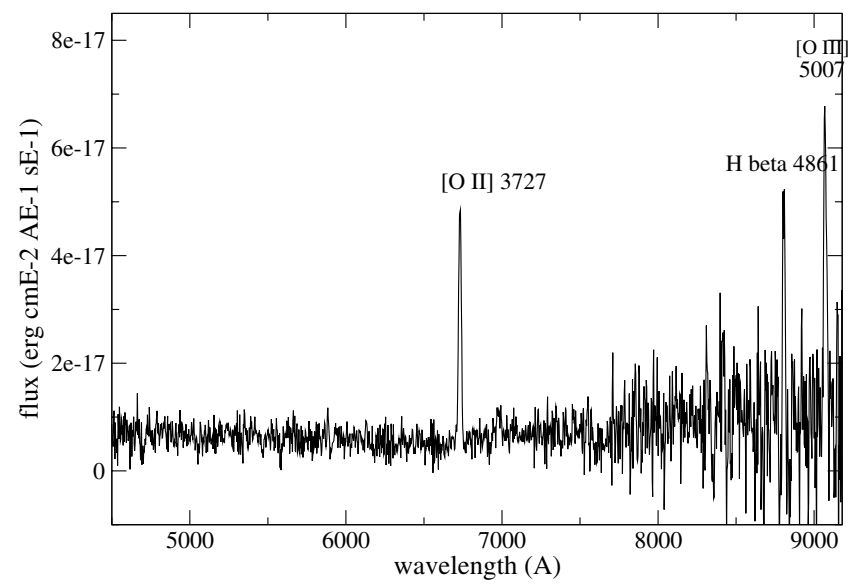

Fig. 6. The optical spectrum of the GRB 051022 host galaxy taken at the 6.0 m BTA (+SCORPIO) on 12 Dec. 2006. No correction for Galactic extinction has been applied. Residuals of sky emission features are visible above $7700 \AA$. The three emission lines detected are consistent with $z=0.809 \pm 0.002$.

assuming a dust-to-gas ratio similar to the Galactic value. Therefore the remaining possibility is that absorbing medium is a giant molecular cloud (GMC) along the line of sight and within the parent host galaxy where such high column densities would be expected. This is also supported by the high absorption column densities (within errors) derived from the HETE-2 and Swift/XRT observations (see also Nakagawa et al. 2006).

\subsection{The nature of the host galaxy}

The optical spectrum of the GRB 051022 host galaxy (Fig. 6) shows one prominent emission line at $6731 \AA$ which we interpreted as [O II] $3727 \AA$ at a redshift $z=0.806 \pm 0.001$. This is confirmed by the additional detection of [O III] $5007 \AA$ at $9071 \AA$ and $\mathrm{H} \beta$ at $8806 \AA$ consistent with $z=0.811$. The [O III] $4946 \AA$ falls within prominent sky lines and could not be accurately determined. The emission lines strengths were measured using a Gaussian fit in IRAF splot. The details are presented in Table 4. No correction for either Galactic extinction or underlying Balmer absorption is applied. This redshift is in agreement with the value $z=0.8$ reported by Gal-Yam et al. (2005). The corresponding luminosity distance is $D_{\mathrm{L}}=5.09 \pm$ $0.20 \mathrm{Gpc}$ (assuming $H_{0}=71 \mathrm{~km} \mathrm{~s}^{-1} \mathrm{Mpc}^{-1}, \Omega_{\mathrm{m}}=0.27$ and $\Omega_{\Lambda}=0.73$ ), from which Rol et al. (2007) derived a collimationcorrected energy released in gamma-rays for GRB 051022 of $(8-18) \times 10^{50} \mathrm{erg}$.

We derive a lower limit on the star formation rate $(S F R)$ from the strength of the [O II] line, applying the calibration of Kennicutt (1998), SFR $\left(M_{\odot} / \mathrm{yr}\right)=(1.4 \pm 0.4) \times 10^{-41} L_{[\mathrm{OII}]}$. Using the measured line luminosity as a lower limit implies $S F R\left(M_{\odot} / \mathrm{yr}\right)>3.2 M_{\odot} / \mathrm{yr}$ and the $S F R$ becomes $S F R\left(M_{\odot} / \mathrm{yr}\right)=$ (48 \pm 3$) M_{\odot} / \mathrm{yr}$ when the intrinsic $A_{V}=1.0$ is taken into account.

Another independent measurement for the $S F R$ can be obtained from the UV continuum at $2800 \AA$, which is produced by massive stars in the galaxy. Using the host galaxy SED, and the Kennicutt (1998) estimator, $\left(M_{\odot} / \mathrm{yr}\right)=1.4 \times$ $10^{-28} L_{v(U V)}\left(\mathrm{erg} \mathrm{s}^{-1} \mathrm{~Hz}^{-1}\right)$, we derive a lower limit for the $\operatorname{SFR}(\mathrm{UV})\left(M_{\odot} / \mathrm{yr}\right) \geq 8 \quad M_{\odot} / \mathrm{yr}$, which becomes $\operatorname{SFR}(\mathrm{UV})\left(M_{\odot} / \mathrm{yr}\right)=59 M_{\odot} / \mathrm{yr}$ when the intrinsic $A_{V}=1.0$ 
Table 3. GRB 051022 host galaxy optical and nIR photometry.

\begin{tabular}{cccccccc}
\hline \hline$U$ & $B$ & $V$ & $R$ & $I$ & $J$ & $H$ & $K$ \\
$22.60 \pm 0.20$ & $22.61 \pm 0.10$ & $21.93 \pm 0.12$ & $21.50 \pm 0.10$ & $20.84 \pm 0.12$ & $20.36 \pm 0.12$ & $19.30 \pm 0.08$ & $18.15 \pm 0.23$ \\
\hline
\end{tabular}

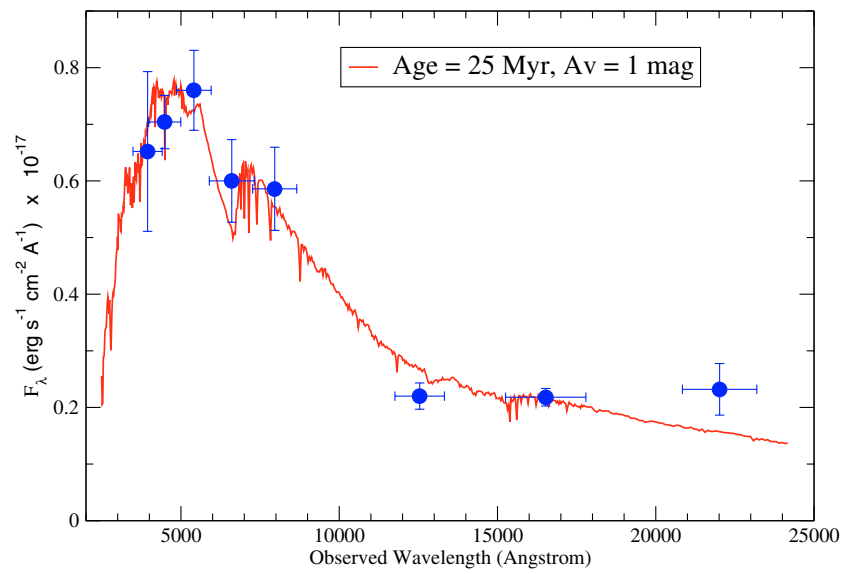

Fig. 7. The SED of the GRB 051022 host galaxy from the $U$ to the $K_{S}$ band. The data are well fit $\left(\chi^{2} /\right.$ dof $\left.=1.04\right)$ by a Im model with considerable extinction, $A_{\mathrm{V}}$ (host) $=1.0 \mathrm{mag}$ and by a starburst episode starting $\sim 25$ Myr ago.

is taken into account, and considering a correction factor for the $2800 \AA$ wavelength.

The detection of [O II] $\lambda 3727, \mathrm{H} \beta \lambda 4861$ and [O III] $\lambda 5007$ allowed us to estimate the value of the $R_{23}$ metallicity indicator (Pagel et al. 1979). To calculate $R_{23}$ we assumed a flux ratio of $1 / 3$ of [O III] $\lambda 4959$ with respect to [O III] $\lambda 5007$. Assuming a host galaxy extinction of $A_{\mathrm{V}}=1.0 \mathrm{mag}$, a SMC-like extinction law (Pei 1992), and correcting for the Galactic extinction $(E(B-V)=0.06$, according to Schlegel et al. 1998) we obtained a value of $R_{23}=4.0 \pm 0.3$. However, without detections of other emission lines (like [N II]) we can not break the $R_{23}$ degeneracy, and there are two possible metallicity values. Given that our [O III]/ [O II] flux ratio (once corrected for Galactic and host galaxy extinction) is $\sim 1$, our $R_{23}$ yields $12+\log (\mathrm{O} / \mathrm{H})$ values of $\sim 7.7$ (lower branch) and $\sim 8.7$ (upper branch), respectively (see Fig. 4 of Maier et al. 2004). The metallicity-luminosity relation at medium redshift galaxies $(z=0.64$, Maier et al. 2004) predicts a metallicity around $12+\log (\mathrm{O} / \mathrm{H}) \sim 9.1$ for the bright absolute magnitude of our host $\left(M_{B}=-21.8\right)$, hence supporting the metallicity obtained with the upper $R_{23}$ branch consistent with $Z \sim Z_{\odot}$.

The spectral energy distribution of the GRB 051022 host galaxy is shown in Fig. 7. The restframe colours (and therefore the associated K-corrections) have been obtained based on the HyperZ code (Bolzonella et al. 2000), fitting synthetic SED templates to our $U B V R I J K_{S}$-band magnitudes derived from our own data (see Table 3 and correcting all for the Galactic reddening $E(B-V)=0.06$, Schlegel et al. 1998). At $z=0.809$, this is a rather luminous galaxy, with $M_{B}=-21.8$ (or $3 \times L^{\star}$ adopting $M_{B}=-20.6$ for a $L^{\star}$ galaxy from Schechter 1976).

Using an apparent size of $\sim 1^{\prime \prime}$ in our images, this would correspond to about $8 \mathrm{kpc}$ at $z=0.809$, typical of compact galaxies and therefore it is possible that the GRB 051022 host would be a blue compact galaxy. Its $M_{B}$ value would place it at the top of their luminosity distribution (Bergvall \& Östlin 2002).

The mass of the GRB 051022 can be determined using several methods: i) from the fitting performed with HyperZ, we get
Table 4. Emission lines detected in the GRB 051022 host galaxy.

\begin{tabular}{lccc}
\hline \hline Line ID & $\lambda_{\text {obs }}$ & $z$ & Flux $\left(10^{-16} \mathrm{erg} \mathrm{cm}^{-2} \mathrm{~s}^{-1}\right)$ \\
\hline [O II] $\lambda 3727$ & 6730.7 & 0.806 & $9.4 \pm 0.3$ \\
$\mathrm{H} \beta \lambda 4861$ & 8806.3 & 0.811 & $7.9 \pm 0.6$ \\
{$[\mathrm{O} \mathrm{III]} \lambda 5007$} & 9070.6 & 0.811 & $13.4 \pm 0.5$ \\
\hline
\end{tabular}

Table 5. Host galaxies of dark/grey GRBs.

\begin{tabular}{lccccc}
\hline \hline $\begin{array}{l}\text { GRB } \\
\text { (YYMMDD) }\end{array}$ & Redshift & $\begin{array}{c}A_{V} \text { (host) } \\
(\mathrm{mag})\end{array}$ & $M_{B}$ & $\begin{array}{c}\text { Starburst age } \\
(\mathrm{Myr})\end{array}$ & $\begin{array}{c}S F R \\
\left(M_{\odot} / \mathrm{yr}\right)\end{array}$ \\
\hline 000210 & 0.842 & 0 & -19.0 & $\sim 180$ & $\sim 2$ \\
000418 & 1.118 & 0.12 & -20.6 & $\sim 50$ & $\sim 8$ \\
051022 & 0.809 & 1.0 & -21.8 & $\sim 25$ & $\sim 50$ \\
\hline
\end{tabular}

a lower limit of $>4.3 \times 10^{9} M_{\odot}$; ii) following Bell et al. (2005), a value of $2 \times 10^{10} M_{\odot}$ is derived; and iii) $4 \times 10^{11} M_{\odot}$ from Brinchmann \& Ellis (2000). The large scatter is caused by the asumptions inherent to each method.

How does the GRB 051022 host compare to other dark GRB host galaxies? Table 5 displays several properties for three dark/grey GRB host galaxies: GRB 000210 (Gorosabel et al. 2003a), GRB 000418 (Gorosabel et al. 2003b) and GRB 051022. As it can be seen, the GRB 051022 host galaxy seems to be a luminous galaxy $\left(M_{B}=-21.8\right)$, with a $S F R$ of $\sim 50 M \odot / \mathrm{yr}^{-1}$, among the highest $S F R$ found in $G R B$ host galaxies.

\section{Conclusions}

We have shown multiwavelength observations of the long duration gamma-ray burst detected by HETE-2 (GRB 051022) between $2.5 \mathrm{~h}$ and $\sim 30$ days after the event. Although a mm transient was found, no optical/nIR afterglow emission has been detected at the position of the X-ray afterglow detected by Swift.

The X-ray spectrum show evidence of absorption by neutral gas with $N_{\mathrm{H}, \mathrm{X} \text {-ray }}=0.85 \times 10^{22} \mathrm{~cm}^{-2}$ (in the observer frame), but ISM absorption by dust in the host galaxy at $z=0.809$ cannot fully account for the non-detection of the optical (and nIR) afterglow. Therefore we are left with the possibility that that the afterglow was extinguished along the line of sight by a giant Molecular cloud (GMC) where the $N_{\mathrm{H}, \mathrm{X} \text {-ray }}$ values are typical to the one found here and therefore no optical/nIR afterglow could be detected as $A_{\mathrm{V}} \sim 50 \mathrm{mag}\left(A_{\mathrm{K}} \sim 6 \mathrm{mag}\right)$.

GRB 051022 is one of the most intense dark GRBs detected in the afterglow era and its host galaxy is, unlike, most of the GRB hosts, brighter than $L^{*}$. We have also shown that the host galaxy is forming stars at a significant rate and derived a $S F R$ $(\mathrm{UV}) \geq 8 M_{\odot} / \mathrm{yr}$. We foresee that this galaxy will be detected at sub-mm wavelengths as was the case for the host galaxies of GRB 000210 and GRB 000418 (Berger et al. 2003).

The synergy between missions like HETE-2 and Swift, the latter being able to be repointed and detect the X-ray afterglow, will allow to study the population of dark GRBs to determine if extinction in the host galaxy (as we have argued in the case for 
GRB 051022) is the reason that about one-half of afterglows are beyond the reach of current optical telescopes.

Note added in proof. The flaring activity seen in our $\mathrm{mm}$ data is concurrent with that at $4.8 \mathrm{GHz}$ reported by Rol et al. (2007). Interstellar scintillation (Rol et al. 2007), weak at $90 \mathrm{GHz}$, is unlikely to provide a common explanation for both. A sudden release of energy appears to be indicated, as we suggest in Sect. 3.2.

Acknowledgements. We thank the anonymous referee for useful comments. This work is based partly on observations carried out with the IRAM Plateau de Bure Interferometer. IRAM is supported by INSU/CNRS (France), MPG (Germany) and IGN (Spain). This work has partially made use of data products from the Two Micron All Sky Survey (2MASS), which is a joint project of the Univ. of Massachusetts and the Infrared Processing and Analysis Center/California Institute of Technology, funded by the National Aeronautics and Space Administration and the National Science Foundation. This research has also been partially supported by the Ministerio de Ciencia y Tecnología under the programmes AYA2004-01515 and ESP2005-07714-C03-03 (including FEDER funds). SMB acknowledges the support of the European Union through a Marie Curie Intra-European Fellowship within the Sixth Framework Program.

\section{References}

Arnaud, K. A. 1996, ASP Conf. Ser., 101, 17

Bell, E. F., Papovich, C., Wolf, C., et al. 2005, ApJ, 625, 23

Berger, E., \& Wyatt, P. 2005, GCN Circ., 4148

Berger, E., Cowie, L. L., \& Kulkarni, S. R., et al. 2003, ApJ, 588, 99

Bergvall, N., \& Östlin, G. 2002, A\&A, 390, 891

Bolzonella, M., Miralles, J.-M., \& Pelló, R. 2000, A\&A, 363, 476

Brinchmann, J., \& Ellis, R. S. 2000, ApJ, 536, 177

Burenin, R., Denisenko, D., Pavlinsky, M., et al. 2005, GCN Circ., 4181

Butler, N. R., Ricker, G. R., Lamb, D. Q., et al. 2005, GCN Circ., 4170

Campana, S., Romano, P., Covino, S., et al. 2006, A\&A, 449, 61

Castro-Tirado, A. J., de Ugarte Postigo, A., Bihain, G., et al. 2005, GCN Circ., 4143

Cameron, P., \& Frail, D. 2005, GCN Circ., 4154

Dickey, J. M., \& Lockman, F. J. 1990, ARA\&A, 28, 215
Djorgovski, S. G., Frail, D. A., Kulkarni, S. R., et al. 2001, ApJ, 562, 654 Feroci, M., Antonelli, L. A., Guainazzi, M., et al. 1997, A\&A, 332, L29 Filliatre, P., Covino, S., D’Avanzo, P., et al. 2006, A\&A, 448, 971

Fynbo, J., Jensen, B. L., Gorosabel, J., et al. 2001, A\&A 369, 373 Galama, T. J., \& Wijers, R. A. M. J. 2001, ApJ, 549, L209

Gal-Yam, A., Berger, E., Fox, D. B., et al. 2005, GCN Circ., 4156

Gorosabel, J., Castro-Tirado, A. J., Wolf, C., et al. 1998, A\&A, 339, 719

Gorosabel, J., Christensen, L., Hjorth, J., et al. 2003a, A\&A, 400, 127

Gorosabel, J., Klose, S., Christensen, L., et al. 2003b, A\&A, 409, 123

Gorosabel, J., Lund, N., Brandt, S., Westergaard, N. J., \& Castro Cerón, J. M. 2004, A\&A, 427, 87

Jakobsson, P., Hjorth, J., Fynbo, J. P. U., et al. 2004, ApJ, 617, L21

Henden, A. A. 2005, GCN Circ., 4184

Hurley, K., Cline, T., Mitrofanov, I., et al. 2005, GCN Circ., 4139

Kennicutt, R. C. 1998, ARA\&A, 36, 189

Lamb, D. Q. 2001, in Gamma-ray Bursts in the Afterglow Era, ed. E. Costa, F. Frontera, \& J. Hjorth (Berlin, Springer), 297

Maier, C., Meisenheimer, K., \& Hippelein, H. 2004, A\&A, 418, 475

Nakagawa, Y. E., Yoshida, A., Sugita, S., et al. 2006, PASJ, 58, L35

Nysewander, M., Cypriano, E., LaCluyze, A., et al. 2005, GCN Circ., 4152

Olive, J.-F., Ricker, G., Atteia, J.-L., et al. 2005, GCN Circ., 4131

Pagel, B. E. J., Edmunds, M. G., Blackwell, D. E., et al. 1979, MNRAS, 189, 95

Pei, Y. C. 1992, ApJ 395, 130

Piro, L., Frail, D. A., Gorosabel, J., et al. 2002, ApJ, 577, 680

Predehl, P., \& Schmitt, J. H. M. M. 1995, A\&A, 293, 889

Racusin, J., Burrows, D., Gehrels, N., et al. 2005a, GCN Circ., 4141

Racusin, J., Kennea, J., Burrows, D., et al. 2005b, GCN Circ., 4169

Ricker, G. 2005, priv. comm

Rol, E., Wijers, R. A. M. J., Kouveliotou, C., Kaper, L., \& Kaneko, Y. 2005, ApJ, 624,868

Rol, E., van der Horst, A., Wiersema, K., et al. 2007, ApJ, accepted [arXiv:astro-ph/0706.1518]

Sari, R., Piran, T., \& Narayan, R. 1998, ApJ 497, L17

Schaefer, B. E., et al. 2005, GCN Circ., 4132

Schechter, P. 1976, ApJ 203, 297

Schlegel, D. J., Finkbeiner, D. P., \& Davis, M. 1998, ApJ, 500, 525

Tanaka, K., Ricker, G., Atteia, J.-L., et al. 2005, GCN Circ., 4137

Taylor, G. B., Frail, D. A., Kulkarni, S. R., et al. 2000, ApJ, 502, L115

Torii, K., et al. 2005, GCN Circ., 4130

van der Horst, A. J., Rol, E., \& Wijers, R. A. M. J. 2005, GCN Circ., 4158

Vaughan, S., Goad, M. R., Beardmore, A. P., et al. 2006, ApJ, 638, 920 